\title{
Evaluación de las necesidades de los familiares de personas afectadas de Daño Cerebral Adquirido mediante el Cuestionario de Necesidades Familiares
}

\author{
Elena López de Arroyabe-Castillo* y Esther Calvete \\ Universidad de Deusto
}

\begin{abstract}
Resumen: El presente estudio tuvo como objetivo adaptar a población española el Cuestionario de Necesidades Familiares (FNQ; Kreutzer, 1988). Este instrumento fue desarrollado para evaluar las percepciones de los familiares de sus propias necesidades después de que un familiar sufriese un Daño Cerebral Adquirido (DCA). El cuestionario incluye seis tipos de necesidades: Necesidad de Información Médica/Salud, Necesidad de Apoyo Emocional, Necesidad de Apoyo Instrumental, Necesidad de Apoyo Profesional, Necesidad de Apoyo Comunitario y Necesidad de Implicación en el Tratamiento y Cuidado. En el estudio participaron 223 familiares de personas afectadas de DCA. El análisis factorial confirmó la estructura original de 6 factores y los coeficientes de consistencia interna fueron buenos. Los resultados muestran además que las necesidades de información son las valoradas como más importantes y a la vez son las más satisfechas. En contraste, las necesidades de apoyo emocional y profesional son las menos satisfechas.
\end{abstract}

Palabras Clave: Daño cerebral adquirido (DCA); Rehabilitación; Necesidades familiares; Apoyo social.
Title: Assessing the needs of the families of persons affected by Acquired Brain Injury with the Family Needs Questionnaire.

Abstract: The present study aimed to adapt the Family Needs Questionnaire (FNQ; Kreutzer, 1988) to the Spanish population. This instrument was developed to assess perceptions of the families of their own needs af ter Acquired Brain Injury (ABI) of a relative. The questionnaire includes 6 factors: Need for Health Information, Need for Emotional Support, Need for Instrumental Support, Need for Professional Support, Need for a Support Network and Need for Involvement with Care. 223 family members of persons with ABI participated in this study. Factor analysis confirmed the original 6 factor structure and internal consistency coefficients were good. The results also show that information needs are considered as the most important and satisfied. In contrast, the needs of professional and emotional support are the least satisfied.

Key words: Acquired brain Injury (ABI); Rehabilitation; Family needs; Social support.

\section{Introducción}

La incidencia anual de daño cerebral adquirido (DCA) aumenta continuamente debido a la mayor eficacia de los cuidados críticos y de resucitación en unidades de cuidados intensivos. Constituye un problema de salud prevalente y una de las principales causas de discapacidad permanente (Chan, Parmenter y Stancliffe, 2009; Defensor del Pueblo, 2005). De ahí que algunos autores se hayan referido a este contraste entre éxito en la resucitación y las dificultades de vida posteriores como el "fracaso del éxito" (Muñoz Céspedes, Paúl, Pelegrín y Tirapu, 2001, p.351). Las consecuencias son muy complejas y discapacitantes, presentando estos enfermos problemas muy severos en diferentes áreas (e.g. motoras, sensoriales y cognitivas), con las consiguientes repercusiones sobre la vida diaria, laboral, social, recreativa, vocacional y económica, tanto para ellos como para sus familias.

Alberdi, Iriarte, Gorostidi, Murgialdai y Marco (2009) señalan que existe una ruptura en la coordinación institucional sanitaria, entre la fase aguda y subaguda propias del tratamiento hospitalario inicial de las lesiones cerebrales y su seguimiento posterior. Esta paradoja se concreta en la expresión familiar "se salva la vida, ¿y ahora?" (Defensor del pueblo, 2005, p.11), la cual sugiere el impacto que el DCA puede tener para la familia de la persona afectada. De hecho, el DCA puede convertirse en uno de los sucesos más devastadores que una familia puede experimentar (Deboskey, Calub, Burton y Morin, 1996; Degeneffee y Olney, 2010). Algunas investigaciones han llegado a plantear que muchas familias soportan un nivel de estrés incluso mayor que la que

* Dirección para correspondencia [Correspondence address]: Elena López de Arroyabe. Facultad de Psicología y Educación de la Universidad de Deusto. Apdo 1, 48080 Bilbao (España).

E-mail: elena.lpzdearroyabe@deusto.es sufren los propios pacientes (Brooks, 1991; Gómez Pastor, 2008), al tener una mayor conciencia de la situación. Los reajustes necesarios en el funcionamiento de las familias transcurren en un período de tiempo prolongado. Los afectados por un DCA y sus familias pueden necesitar ayuda profesional para mantener una calidad psicosocial de vida razonable, incluso más de una década después de la lesión (Hoofien, Gilboa, Vakil y Donovick, 2001; Ponsford, Sloan y Snow, 1995; Thomsen, 1984; Verhaeghe, Delfloor y Grypdonck, 2005).

El estrés y la carga del familiar tienden a ser crónicos (Masel y Dewitt, 2010) y en muchos casos no sólo no disminuyen sino que pueden incluso aumentar con el paso del tiempo (Brooks, Campsie, Symington, Beattie y McKinlay, 1986; Douglas y Spellacy, 1996; Fernández Guinea y Muñoz Céspedes, 1997; Gillen, Tennen y Affleck, 1998; Hawley, Ward, Magnay y Long, 2003; Jhonson y McCown, 1997; Kreutzer, Marwitz y Kepler, 1992; Wade et al., 2002) marcando realmente la diferencia entre el DCA y otras experiencias traumáticas, en las que los niveles tienden a decrecer.

El apoyo social tiene una relación directa con el funcionamiento familiar tras un DCA y es el moderador del estrés del cuidador con más poder (Ergh, Rapport, Coleman y Hanks, 2002; Verhaeghe et al. 2005). Mauss-Clum y Ryan (1981) fueron de los primeros en estudiar empíricamente las necesidades familiares de apoyo en el DCA y resaltar la importancia de incluir al familiar activamente en el proceso rehabilitador. En su estudio, encontraron que los familiares valoraban muy alto la necesidad de comunicarse con los profesionales acerca de la recuperación tanto a corto como a largo plazo de su familiar afectado, así como la necesidad de apoyo emocional, información sobre recursos comunitarios y apoyo financiero. Poco después surgieron otros estudios que acentuaron la importancia de atender las necesidades familiares lo antes posible tras la lesión (Campbell, 1988, Mathis, 
1984). En este contexto Kreutzer (1988) elaboró el Cuestionario de Necesidades Familiares en DCA (FNQ). Se trata de un instrumento estandarizado que aporta al profesional información sobre las necesidades del familiar y en qué grado han sido atendidas o no, proporcionando un mejor acercamiento a los cambios que se den en el proceso dinámico de la rehabilitación e integración social. De hecho, pasar el cuestionario antes de la terapia facilita y guía la intervención, haciéndola más efectiva. La mayoría de los ítems se seleccionaron a partir de una revisión de la bibliografía existente sobre las reacciones de la familia ante el daño cerebral (Campbell, 1988; Leske, 1986; Mathis, 1984; Mauss-Clum y Ryan, 1981; Molter, 1979; Norris y Grove, 1986). El resto de los ítems se obtuvieron a partir de la evaluación de las necesidades que los familiares expresaban con más frecuencia en las entrevistas clínicas. En la versión final se tuvo en cuenta tanto a los pacientes hospitalizados como a los dados de alta. El tiempo necesario para completar el cuestionario es de 10-15 minutos.

El FNQ evalúa necesidades que pueden aparecer tanto durante la rehabilitación aguda como tras el alta. Serio, Kreutzer y Witol (1997) mediante análisis factorial identificaron seis factores: Necesidad de Información Médica/Salud (disponer de información clara y sincera sobre los problemas de salud de la persona afectada), Necesidad de Apoyo Emocional (protección y amparo de su estado de ánimo), Necesidad de Apoyo Instrumental (ayuda tangible para el hogar, descanso y "respiro" personal), Necesidad de Apoyo Profesional (servicios de rehabilitación, recursos financieros/legales y profesionales que ejerzan su profesión con capacidad y aplicación), Necesidad de una red de Apoyo Comunitario (comprensión proveniente de familia, amigos y compañeros y seguimiento profesional) y Necesidad de Implicación en el Tratamiento y Cuidado (participar en la rehabilitación).

El objetivo de este estudio fue adaptar el Cuestionario de Necesidades Familiares a población española y evaluar las necesidades de los familiares de personas afectadas por un DCA en la fase post-hospitalaria (vuelta a casa y permanencia). De esta manera se quiso identificar áreas fuertes y puntos de mejora en cuanto a las necesidades de los familiares.

\section{Método}

\section{Participantes}

Las personas participantes en el presente estudio fueron miembros de Asociaciones de afectados y familiares que pertenecen a la Federación Española de Daño Cerebral Sobrevenido (FEDACE) y familiares de personas con DCA atendidas en un centro de tratamiento privado. Se enviaron 809 cuadernillos. El total de familiares que contestaron las pruebas fue de $223(27.5 \%)$, de los que la mayoría procedieron de FEDACE (97.31\%). El promedio de tiempo que llevaban asociados a FEDACE fue de 4.54 años $(D T=3.07)$, siendo el tiempo promedio transcurrido desde la lesión cerebral hasta la realización del estudio de 8.15 años. El nivel de participación obtenido puede considerarse satisfactorio, dada la longitud y complejidad de los instrumentos utilizados así como el cansancio que el familiar puede acumular en periodos de tiempo muy largos de cuidado.

Las edades de los participantes oscilaron entre 20 y 77 años $($ Edad Media $=49.86, D T=12.63)$. Un $83 \%$ vivían con la persona afectada y el $40 \%$ le dedicaban 60 horas semanales (acompañando o cuidando de él/ella), es decir una media diaria de más de 8 horas. El 26.9\% eran varones y el $72.2 \%$ mujeres. La Tabla 1 describe la muestra en cuanto a parentesco con la persona afectada, estudios y tipo de convivencia. Tal y como puede observarse la mayoría son progenitores y cónyuges que conviven con la persona afectada y que están en activo, trabajando por cuenta ajena o en las labores del hogar.

Tabla 1. Parentesco con la persona afectada.

\begin{tabular}{lll}
\hline El familiar es su & $\mathrm{n}$ & $\%$ \\
\hline Padre / Madre & 85 & 38.1 \\
Cónyuge & 75 & 34.1 \\
Hijo/a & 36 & 15.9 \\
Hermano/a & 24 & 11.1 \\
Otro & 3 & 1.4 \\
\hline Estudios & & \\
\hline Cultura General & 53 & 23.8 \\
Bachiller Elemental & 54 & 24.2 \\
FP o Bachiller Superior & 58 & 26.0 \\
Diplomatura & 35 & 15.7 \\
Licenciatura & 21 & 9.4 \\
\hline Ocupación del familiar & & \\
\hline Trabaja & 99 & 44.4 \\
Ama de casa & 71 & 31.8 \\
Busca empleo & 14 & 6.3 \\
Jubilado/ a & 30 & 13.5 \\
Estudiante & 1 & 0.4 \\
Autónomo/a & 3 & 1.3 \\
En excedencia & 2 & 0.9 \\
Baja laboral & 2 & 0.9 \\
\hline Tipo de Convivencia & & \\
\hline Convive & 185 & 83.0 \\
No convive & 37 & 16.6 \\
Sólo fines de semana & 1 & 0.5 \\
\hline
\end{tabular}

\section{Instrumento de medida}

Las necesidades del familiar fueron evaluadas con el FNQ (Kreutzer, 1988). El FNQ incluye 40 ítems que reflejan diversas necesidades que pueden aparecen tanto durante la rehabilitación aguda como tras el alta. Cada pregunta contiene dos partes. En la primera, se indica en qué medida es importante esa necesidad para el familiar, puntuando en una escala de 4 puntos, desde "no es importante" hasta "muy importante". En la segunda, el familiar indica en qué medida esa necesidad se ha satisfecho: "si", "parcialmente", "no".

Serio y Kreutzer (1992) y Serio et al. (1997), en sendos estudios analíticos de factores, identificaron seis factores. Serio y Kreutzer (1992), refirieron cuatro factores conceptual- 
mente más fuertes (Necesidad de Información Médica/Salud, Necesidad de Apoyo Emocional, Necesidad de Apoyo Instrumental y Necesidad de Apoyo Profesional) y dos factores con menor apoyo teórico (Necesidad de Apoyo Comunitario y Necesidad de una Implicación en el Tratamiento y Cuidado del Paciente). El primer factor, Necesidad de Información Médica/Salud, consta de 10 ítems cuyos contenidos se basan en la comunicación al familiar de información (comprensible y sincera) sobre el estado médico, educacional y de rehabilitación de la persona afectada (problemas, cambios, avances, garantía de que se da la mejor atención y se respeta las necesidades/deseos del paciente). El factor de Necesidad de Apoyo Emocional consta de 8 ítems y sus contenidos se basan en la necesidad del familiar de expresar sus sentimientos, miedos/dudas sobre el futuro, mantener la esperanza o estar preparado para lo peor, que los demás y la propia persona afectada entiendan lo difícil que es para él o ella y recibir ánimo para pedir ayuda. El factor de Necesidad de Apoyo Instrumental consta de 6 ítems y sus contenidos se basan en la necesidad de ayuda menajera para el hogar- de otros miembros de la familia, disponer de un "respiro" para poder descansar física y psicológicamente, estar con amigos y atender sus propias necesidades. El factor de Necesidad de Apoyo Profesional consta de 5 ítems y sus contenidos se basan en la necesidad de contar con un profesional que indique pautas de actuación ante los problemas de la persona afectada, pronóstico y disponer de suficientes recursos para el paciente y la familia. El factor de Necesidad de Apoyo Comunitario consta de 5 ítems y sus contenidos se basan en la necesidad de disponer de un profesional de asesoramiento o servicios cuando el paciente lo necesite, que otros familiares amigos, jefes o profesores entiendan los problemas del paciente y poder hablar de sus sentimientos respecto al paciente con amigos/familia. Por último, el factor de Necesidad de Implicación en el Tratamiento y Cuidado consta de 3 ítems y sus contenidos se basan en la necesidad del familiar de ser tenido en cuenta y participar en la planificación del tratamiento y su aplicación. El FNQ ha obtenido buenos indicadores de consistencia interna (Kreutzer, Serio y Begquist, 1994). El coeficiente alfa para las 6 subescalas oscila entre .78 y .89 .

Este cuestionario ha sido traducido empleando un método de retroalimentación al castellano por las autoras de este proyecto y tras contar con la autorización del autor para su uso en el presente estudio.

\section{Procedimiento}

Se contactó con las Juntas y/o equipos de profesionales de todas las Asociaciones federadas en FEDACE y con un centro de tratamiento privado, solicitando su colaboración en la investigación, enviándoles un breve resumen con las líneas generales de la investigación y un modelo de carta de presentación para el familiar a quien se solicitaba su colaboración. Los cuestionarios fueron contestados anónimamente a fin de garantizar la confidencialidad y la sinceridad en las respuestas.

\section{Resultados}

\section{Estructura factorial del FNQ}

Se realizó un análisis factorial confirmatorio basado en el método de máxima verosimilitud con el programa LISREL 8.8 , en el que se probó la hipótesis de que un modelo de seis factores (apoyo informativo, emocional, instrumental, profesional, comunitario y de implicación) resultaría en una adecuada explicación de las necesidades comunicadas por los familiares. El modelo fue el mismo que obtuvieron Serio y Kreutzer (1992) y Serio et al. (1997) y se basó únicamente en los 37 ítems que se agruparon en los seis factores en los estudios previos y en las puntuaciones de importancia concedida a cada necesidad.

Siguiendo las recomendaciones de diversos autores (e.g. Hoyle y Panter, 1995; Hu y Bentler, 1999) se evaluó la adecuación del ajuste mediante el CFI (Comparative Fit Index), el NNFI (Nos-Normed Fit Index) y el SRMR (Standardized Root Mean Square Residual). Generalmente los valores de NNFI y CFI de 90 o mayores reflejan un buen ajuste. Un valor SRMR de hasta .08 indica un error razonable en la estimación. Siguiendo estos criterios, los indicadores de ajuste fueron adecuados, $\chi^{2}(613, N=223)=1688$, SRMR $=.08$, $\mathrm{NNFI}=.96, \mathrm{CFI}=.96$.

La Tabla 2 recoge los coeficientes Lambda de cada ítem en su factor correspondiente. Tal y como puede observarse todos los coeficientes fueron adecuados y en todos los casos los valores fueron significativamente diferentes de cero ( Valor absoluto de $t$ mayor que 1.96). Los coeficientes alpha fueron buenos, tal y como se recoge en la Tabla 3.

Tabla 2. Coeficientes Lambda-Y de los ítems del FNQ en los factores del FNQ

\begin{tabular}{|c|c|}
\hline \multirow{2}{*}{\multicolumn{2}{|c|}{ Necesidad de Información Médica/Salud }} \\
\hline & \\
\hline 1. Poder comprobar que el personal médico, educacional o de rehabilitación respeta las necesidades o deseos del paciente. & 0.71 \\
\hline 4. Que me comuniquen sobre todos los cambios que se produzcan en el estado médico del paciente. & 0.87 \\
\hline 5. Que me garanticen que se da al paciente la mejor atención médica posible. & 0.88 \\
\hline 6. Que los profesionales me den explicaciones en términos que los pueda entender. & 0.94 \\
\hline 7. Que respondan a mis preguntas sinceramente. & 0.92 \\
\hline $\begin{array}{l}\text { 11. Disponer de una información completa sobre la atención médica sobre heridas traumáticas (a saber: medicación, inyeccio- } \\
\text { nes o cirugía). }\end{array}$ & 0.58 \\
\hline
\end{tabular}

12. Disponer de una información completa sobre los problemas físicos del paciente (a saber: debilidad, dolores de cabeza, ma- 0.60 
reos, problemas de visión o de movimiento).

13. Disponer de una información completa sobre los problemas mentales del paciente (a saber: confusión, memoria o comuni- 0.64 cación).

14. Disponer de una información completa sobre problemas de drogas o alcohol y sobre su tratamiento.

18. Tener información sobre el avance del paciente respecto a la rehabilitación o a la educación.

Necesidad de Apoyo Emocional

29. Que la persona en cuestión (la persona afectada) entienda lo difícil que es para mí.

30. Que mi pareja o amigos entiendan lo difícil que es para mí.

34. Hablar de mis sentimientos sobre el paciente con alguien que haya pasado por la misma experiencia.

36. Que me tranquilicen en el sentido de que es normal tener fuertes sentimientos negativos respecto al paciente.

37. Ayuda para superar mis dudas y miedos sobre el futuro.

38. Ayuda para mantener la esperanza sobre el futuro del paciente.

39. Ayuda para estar preparado / a para lo peor.

40. Que se me anime a pedir a los demás que me echen una mano.

Necesidad de Apoyo Instrumental

22. Disponer de ayuda para las tareas de la casa (a saber: hacer la compra, la limpieza, la cocina, etc.)

23. Disponer de la ayuda de otros miembros de la familia para cuidar al paciente.

24. Dormir y descansar lo suficiente.

25. Tomarme un descanso de mis problemas y responsabilidades.

26. Estar con mis amigos.

27. Prestar atención a mis propias necesidades, trabajo o intereses.

\section{Necesidad de Apoyo Profesional}

16. Que se me comunique cuánto se espera que duren cada uno de los problemas del paciente.

17. Que se me indique lo que hacer cuando el paciente se disgusta o actúa de manera extraña.

19. Contar con ayuda para decidir en qué medida hay que dejar al paciente valerse por sí mismo.

20. Disponer de suficientes recursos para el paciente (a saber: programas de rehabilitación, terapia física, orientación, orienta- 0.84 ción laboral).

21. Disponer de suficientes recursos para mí o para la familia (a saber: orientación financiera o legal, un descanso, orientación, 0.74 atención profesional, enfermería, atención de día).

Necesidad Apoyo Comunitario

9. Disponer de un profesional al que pueda recurrir para pedirle consejo o servicios cuando el paciente necesite ayuda. 0.62

31. Que los otros miembros de la familia entiendan los problemas del paciente.

32. Que los amigos del paciente entiendan sus problemas.

33. Que el jefe, colegas o profesores del paciente entiendan sus problemas.

35. Hablar de mis sentimientos respecto al paciente con amigos o con la familia. 0.76

Necesidad de Implicación en el Tratamiento y Cuidado

2. Que me comuniquen diariamente lo que se está haciendo con o para el paciente.

3. Dar mis opiniones diariamente a otras personas que participen en el cuidado, rehabilitación o educación del paciente

Tabla 3. Coeficientes de correlación entre las escalas de importancia de necesidades percibidas en la fase de vuelta a casa/permanencia y coeficientes de consistencia interna

\begin{tabular}{lllllll}
\hline & 1 & 2 & 3 & 4 & 5 & 6 \\
\hline 1. Información & & & & & & \\
2. Emocional & .40 & & & & & \\
3. Instrumental & .44 & .61 & & & & \\
4. Profesional & .73 & .47 & .57 & & & \\
5. Comunitario & .45 & .73 & .58 & .58 & & \\
6. Implicación & .75 & .39 & .37 & .58 & .38 & \\
Media & 3.43 & 3.15 & 3.28 & 3.52 & 3.41 & 3.17 \\
DT & 0.71 & 0.90 & 0.85 & 0.76 & 0.69 & 0.85 \\
Alpha & .87 & .90 & .88 & .88 & .72 & .76 \\
\hline
\end{tabular}

Nota. Todos los coeficientes de correlación son estadísticamente significativos al nivel $p<.001$

\section{Importancia de las necesidades}

La Tabla 3 presenta las medias, desviaciones típicas y coeficientes de correlación entre las puntuaciones en importancia de las seis dimensiones. La tabla muestra como las dimensiones más importantes son la Necesidad de Informa- ción Médica/Salud y la Necesidad de Apoyo Profesional. En concreto, un análisis por ítems mostró que las 10 mayores puntuaciones en importancia ( 1 a 4) en la fase de vuelta a casa/permanencia se obtuvieron para los ítems: 20 (Disponer de suficientes recursos para el paciente: programas de rehabilitación, terapia física, orientación y orientación laboral, 3.74), 13 (Disponer de una información completa sobre los problemas mentales del paciente: confusión, memoria o comunicación, 3.72), 9 (Disponer de un profesional al que pueda recurrir para pedirle consejo o servicios cuando el paciente necesite ayuda, 3.70), 12 (Disponer de una información completa sobre los problemas físicos del paciente: debilidad, dolores de cabeza, mareos, problemas de visión o de movimiento, 3.63), 7 (Que respondan a mis preguntas sinceramente, 3.62), 31 (Que los otros miembros de la familia entiendan los problemas del paciente, 3.58), 5 (Que me garanticen que se da al paciente la mejor atención médica posible, 3.57), 21 (Disponer de suficientes recursos para mí o para la familia: orientación financiera o legal, un descanso, orienta- 
ción, atención profesional, enfermería, atención de día, 3.55), 6 (Que los profesionales me den explicaciones en términos que los pueda entender, 3.54) y 19 (Contar con ayuda para decidir en qué medida hay que dejar al paciente valerse por sí mismo, 3.54).

Tabla 4. Satisfacción completa de necesidades valoradas como importantes en la vuelta a casa/permanencia: frecuencias absolutas y porcentajes

\begin{tabular}{lccc} 
Tipo de Apoyo & Satisfacción promedio (0 a 2) & $\begin{array}{l}\text { Número de necesidades importantes satis- } \\
\text { fechas completamente (media) }\end{array}$ & $\begin{array}{l}\text { Porcentaje (\%) de necesidades importantes } \\
\text { que han sido satisfechas completamente }\end{array}$ \\
\hline 1. Información & 1.22 & $3.66(0 \mathrm{a} 10)$ & 43.68 \\
2. Emocional & 0.93 & $1.58(0 \mathrm{a} 8)$ & 27.09 \\
3. Instrumental & 0.97 & $1.39(0 \mathrm{a} 6)$ & 28.88 \\
4. Profesional & 0.92 & $1.21(0 \mathrm{a} 5)$ & 27.36 \\
5. Comunitario & 1.04 & $1.32(0 \mathrm{a} 5)$ & 32.58 \\
6. Implicación & 1.22 & $0.94(0 \mathrm{a} 3)$ & 41.29 \\
\hline
\end{tabular}

\section{Satisfacción de las necesidades}

Se calculó el grado de satisfacción con cada una de las necesidades. En la Tabla 4 se presentan los datos correspondientes a la satisfacción promedio de cada factor. Tal y como puede observarse los mayores grados de satisfacción se obtuvieron para las necesidades de Apoyo Informativo y de Implicación en el Tratamiento y Cuidado. La mayor insatisfacción se obtuvo para las necesidades de Apoyo Emocional y Profesional.

Una de las utilidades mayores del FNQ es que permite calcular una serie de indicadores relativos al porcentaje de necesidades importantes que han sido completa o parcialmente satisfechas. De esta manera, en este estudio se obtuvieron los siguientes indicadores: (a) frecuencia absoluta de necesidades importantes (puntuadas como 3 o 4) que han sido satisfechas, y (b) porcentaje de necesidades importantes (puntuadas como 3 o 4) que han sido satisfechas (frecuencia absoluta anterior dividida entre el número de necesidades que han sido valoradas como importantes. La tabla 4 presenta también estos indicadores, mostrando que las necesidades que son en menor medida satisfechas son las referentes al Apoyo Emocional y Profesional.

Un análisis pormenorizado por ítems mostró que las 10 necesidades señaladas como menos satisfechas ( $\left.\begin{array}{lll}0 & \text { a }\end{array}\right)$ en la fase de vuelta a casa/permanencia fueron: 39 (Ayuda para estar preparado/a para lo peor, 0.65), 22 (Disponer de ayuda para las tareas de la casa: hacer la compra, la limpieza, la cocina, etc. 0.65), 25 (Tomarme un descanso de mis problemas y responsabilidades, 0.72), 37 (Ayuda para superar mis dudas y miedos sobre el futuro, 0.74), 32 (Que los amigos del paciente entiendan sus problemas, 0.75), 29 (Que la persona afectada entienda lo difícil que es para mí, 0.75), 21 (Disponer de suficientes recursos para mí o para la familia: orientación financiera o legal, un descanso, orientación, atención profesional, enfermería, atención de día, 0.81), 38 (Ayuda para mantener la esperanza sobre el futuro del paciente, 0.83), 33 (Que el jefe, colegas o profesores del paciente entiendan sus problemas, 0.83) y 40 (Que se me anime a pedir a los demás que me echen una mano, 0.87).

\section{Discusión}

El objetivo de este estudio consistió en adaptar a población española el FNQ (Kreutzer, 1988). El FNQ constituye un instrumento de gran utilidad clínica tal y como lo demuestran los numerosos trabajos sobre su aplicación en muestras de familiares (entre otros, Degeneffe, 2009; KolakowskyHayne, Miner y Kreutzer, 2001; Serio, Kreutzer y Gervasio, 1995; Witol, Sander y Kreutzer, 1996). Por ello, su adaptación a población española contribuye a la identificación de áreas fuertes y puntos de mejora en la situación actual de los familiares de personas con DCA en nuestro ámbito.

Este estudio mostró que las propiedades psicométricas de la versión española del FNQ eran adecuadas. El análisis factorial confirmó la estructura de seis factores identificados previamente por Serio et al. (1997). Estos factores son los siguientes: Apoyo Información Médica/Salud, Apoyo Emocional, Apoyo Instrumental, Apoyo Profesional, Apoyo Comunitario e Implicación en el Tratamiento y Cuidado. La mayoría de los seis factores son consistentes con las clasificaciones del apoyo social propuestas por diversos expertos en el ámbito (e.g. Cohen y Wills, 1985; Uchino, 2004; Wortman, 1984). Por ejemplo, Kreutzer et al. (1994) identificaron que cinco de los seis factores, corresponden a las dimensiones de apoyo social descritas por Wortman (1984). Además, los factores mostraron índices de consistencia interna muy adecuados. A excepción del factor Apoyo Comunitario y del factor Implicación, cuyos coeficientes alpha fueron .72 y .76 respectivamente, los demás coeficientes oscilaron entre .87 y .90 , siendo incluso superiores a los encontrados por Serio et al. (1997).

El FNQ permite obtener un considerable número de indicadores acerca de las necesidades de apoyo de los familiares y su satisfacción, tanto si nos basamos en el análisis de ítems como en el de los factores o escalas.

Desde la perspectiva de las escalas del FNQ destaca que todas las áreas obtienen puntuaciones promedio en importancia percibida superiores a 3, indicando que todas estas áreas son consideradas entre importantes y muy importantes. Mayores oscilaciones se encuentran cuando se compara el grado de satisfacción promedio de cada área. Las mayores puntuaciones en satisfacción se dan para la Necesidad de Información Médica/Salud y la Necesidad de Implicación en el 
Tratamiento y Cuidado y las puntuaciones más bajas se dan para el Apoyo Emocional y el Apoyo Instrumental. Además, si se toman en cuenta los porcentajes de necesidades en cada área que han sido satisfechas plenamente, se observa de forma consistente que el Apoyo de Información Médi$\mathrm{ca} /$ Salud e Implicación son las áreas que obtienen porcentajes mayores y que el Apoyo Emocional, Profesional e Instrumental obtienen los porcentajes menores.

Desde el punto de vista de los ítems, en este estudio se identificaron las necesidades más frecuentemente percibidas como importantes durante el período de vuelta a casa/permanencia. De las 10 necesidades señaladas como más importantes, cinco corresponden a la Necesidad de Información Médica/Salud sobre los problemas neuropsicológicos y físicos del paciente, comprensión y sinceridad en la información y atención garantizada y tres a la Necesidad de Apoyo Profesional (que oriente y paute cómo actuar con el paciente, pronóstico y disponer suficientes recursos para el paciente y la familia). Estas necesidades coinciden en general con las necesidades más frecuentemente percibidas como importantes en otros estudios previos (Kreutzer et al., 1994; Witol et al., 1996), sugiriendo la equivalencia de dichas necesidades en diferentes culturas. Por ejemplo, Arango-Lasprilla et al. (2010) han evaluado las necesidades de cuidadores de personas con un DCA en Colombia y confirman que las necesidades de los familiares colombianos son similares a las obtenidas en los estudios previos anglosajones, con la diferencia de que tienden a estar menos cubiertas.

A pesar de estas similitudes, la cultura puede influir en la disponibilidad e importancia dada al apoyo social. En general, la cultura española es menos individualista que la cultura norteamericana (Oyserman, Coon y Kemmelmeier, 2002). Las familias españolas enfatizan el mantenimiento de relaciones armoniosas y permanentes (Oyserman et al. 2002; Triandis, 1995). Como consecuencia, en nuestra cultura se dedica más tiempo a la familia (Rokach, Moya, Orzeck y Exposito, 2001). Esta diferencia podría influir en la relevancia del apoyo social y en las necesidades específicas de los cuidadores de personas con DCA en nuestro ámbito.

Desde el punto de vista de los ítems, en este estudio también se identificaron las diez necesidades que con mayor frecuencia se señalaban como no atendidas en la fase de vuelta a casa/permanencia. La mitad corresponden a la necesidad de apoyo emocional, incluyendo que se prepare al familiar para lo peor, se le anime a pedir ayuda a los demás, a superar dudas y miedos sobre el futuro y que se sientan entendidos por la persona afectada.

Por último, se identificaron las diez necesidades más frecuentemente señaladas como atendidas adecuadamente en la fase de vuelta a casa/permanencia. Siete de estas necesidades corresponden a la Necesidad de Información Médica/Salud indicando que es una de las necesidades mejor cubiertas. Estos resultados coinciden con los obtenidos por Kreutzer et al. (1994) y Serio et al. (1995), quienes también encontraron que la necesidad de apoyo informativo era percibida la más importante y también la más frecuentemente atendida. Por tanto, estos datos en conjunto sugieren que para el familiar la necesidad más importante es la de información y es la que más demanda. De hecho el familiar recibe una mayor información médica, educacional o rehabilitadora y es menos probable que reciba un apoyo emocional adecuado.

El apoyo informativo es más fácil de demandar y también es más evidente a quien solicitarlo: equipo de profesionales, quienes a su vez se responsabilizan de ello como parte de su trabajo. Es decir, se demanda más y es el que más se atiende. Por contra, el apoyo emocional es el que menos se atiende y proviene de fuentes menos tangibles, amigos, familia cercana/extensa, profesionales, etc. (Bolger, Zuckerman y Kessler, 2000; Cutrona y Russell, 1990; Uchino, 2004).

Por tanto, la información no es lo único que hay que ofrecer aunque sea lo que más se demanda. El familiar está tan centrado en la salud del paciente que olvida la suya propia y en puntuales ocasiones solicita apoyo psicológico y emocional. En un estudio con familiares de personas con DCA se encontró que el $28.3 \%$ de los familiares reciben apoyo de otros familiares y el $18.8 \%$ reciben atención psicológica especializada, datos que reflejan la poca ayuda que recibe el familiar tanto por parte de los profesionales como por su propio entorno (López de Arroyabe, 2006). Atendiendo y satisfaciendo las necesidades del familiar, le capacitamos de tal forma que desarrollará con más eficiencia su labor en el proceso de rehabilitación. Además esto repercutirá en una menor carga por cuidar de la persona afectada, redundando en una mejor calidad de vida para ambos.

\section{Conclusiones}

La necesidad más frecuentemente percibida como importante por el familiar es la de Información Médica/Salud siendo también la necesidad más demandada y la más atendida y asumida por los profesionales. En el DCA las múltiples causas así como las características propias del órgano dañado ocasionan muy diferentes altas hospitalarias con pronósticos inciertos y evoluciones muy diversas. Por ello, resulta una necesidad básica y continua conocer la situación en cada momento desde una perspectiva médica, rehabilitadora y educacional. El resto de las necesidades se cimentarán sobre dicho pilar.

La necesidad señalada más frecuentemente como no atendida es la de apoyo emocional. El hecho de que el familiar no sea o no se sienta apoyado emocionalmente interfiere negativamente en sus relaciones con los profesionales, pudiendo repercutir en la recuperación de la persona afectada. Además, el familiar está tan centrado en la salud del paciente que olvida la suya propia y en puntuales ocasiones solicita apoyo psicológico y emocional. El familiar y la persona afectada van a precisar un seguimiento de larga duración y ayuda de instituciones correspondientes tanto al ámbito sanitario como social. Los diferentes profesionales que acompañen también al familiar en este recorrido, desde la fase aguda hasta la crónica, deberían tener en cuenta que la satisfacción del apoyo emocional es la variable que más impacto va a te- 
ner en la reducción de sus síntomas psicológicos (Calvete y López de Arroyabe, 2012). Estos resultados tienen relevancia clínica y pueden ser útiles en el diseño de planes de intervención con familiares. Dichos programas deberían promover el apoyo más básico cuando la situación se percibe como no controlable: el apoyo emocional, autocuidado, compañía y apoyo instrumental, fomentando el desarrollo de recursos y redes sociales que puedan cubrir dichas necesidades.

\section{Referencias}

Alberdi, F., Iriarte, M., Gorostidi, A., Murgialdai, A. y Marco, P. (2009). Puesta al día en Medicina Intensiva: Neurointensivismo Pronóstico de las secuelas tras la lesión cerebral. Medicina Intensiva, 33(4), 171-181.

Arango-Lasprilla, J.C., Quijano, M.C., Aponte, M., Cuervo, M.T., Nicholls, E. Rogers, H.L. et al. (2010). Family needs in caregivers of individuals with traumatic brain injury from Colombia, South America. Brain Injury, 24, 7-8: 1017-1026.

Bolger, N., Zuckerman, A. y Kessler, R. C. (2000). Invisible support and adjustment to stress. Journal of Personality \& Social Psychology, 79, 953-961.

Brooks, N., Campsie L., Symngton, C., Beattie, A. y McKinlay, W. (1986). The five year outcome of severe blunt head injury: a relative's view. Journal of Neurology, Neurosourgery and Psychiatry, 49, 764-770.

Brooks, N.D. (1991). The head injury family. Journal of Clinical and Experimental Neuropsychology, 13, 155-188.

Calvete, E. y López de Arroyabe, E. (2012). Depressive symptoms and grief in Spanish family caregivers of persons with Traumatic Brain Injury: The role of social support and coping. Brain Injury, 26(6), 834-843.

Campbell, C. (1988). Needs of relatives and helpfulness of support groups in severe head injury. Rebabilitation Nursing, 13 ,320-325.

Cohen, S. y Wills, T. A. (1985). Stress, social support, and the buffering hypothesis. Psychological Bulletin, 98(2), 310-357.

Cutrona, C. E. y Russell, D. W. (1990). Type of social support and specific stress: Toward a theory of optimal matching. En B. R. Sarason, I. G. Sarason, y G. R. Pierce (Eds.), Social support: An interactional view (pp. 319-366). New York: John Wiley.

Chan, J., Parmenter, T. y Stancliffe, R. (2009). The impact of traumatic brain injury on the mental health outcomes of individuals and their family carers. Advanced in Mental Health, 8(2), 155-164.

Deboskey, D., Calub, C., Burton, J. y Morin, K. (1996). Life after brain injury: Who Am I? Tampa general Rehabilitation Center, Florida. HDI Publishers.

Defensor del Pueblo (2005). Daño Cerebral Sobrevenido en España: Un acercamiento Epidemiológico y Sociosanitario. Informe del Defensor del Pueblo. Madrid.

Degeneffe, Ch. (2009). The Rehabilitation Needs of Adult Siblings of Persons With Traumatic Brain Injury: A Quantitative Investigation. Australian Journal of Rehabilitation Counselling, 15(1), 12-27.

Degeneffe, Ch. y Olney, M. (2010). 'We are the forgotten victims': Perspectives of adult siblings of persons with traumatic brain injury. Brain Inju$r y, 24(12), 1416-1427$

Douglas, J.M. y Spellacy, F.J. (1996). Indicators of long-term family functioning following severe traumatic brain injury in adults. Brain Injury, 10, 819-839.

Ergh, T., Rapport, L., Coleman, R. y Hanks, R. (2002). Predictors of caregiver and family functioning following traumatic brain injury: Social support moderates caregiver distress. Journal of Head Trauma Rehabilitation, 17(2), 155-74.

Fernández Guinea, S. y Muñoz Céspedes, J.M. (1997). Las familias en el proceso de rehabilitación de las personas con daño cerebral sobrevenido. Psicologia.com 1,1.

Gillen, R., Tenneh, H. y Affleck, G. (1998). Distress, depressive symptoms and depressive disorder among caregivers of patients with brain injury. Journal of Head Trauma Rehabilitation, 13, 31-43.

Gómez Pastor, I. (2008). El daño cerebral sobrevenido: un abordaje transdisciplinar dentro de los servicios sociales. Intervención Psicosocial, 17(3), 237-244.
En este contexto el FNQ se presenta como un instrumento útil en la detección de necesidades de las familias de personas afectadas con DCA. En particular, el análisis de los ítems permite identificar áreas fuertes (necesidades importantes que son señaladas como satisfechas) y puntos de mejora (necesidades importantes que son señaladas como insatisfechas) que orienten las intervenciones.
Hawley, C., Ward, A., Magnay, A. y Long, J. (2003). Parental stress and burden following traumatic brain injury amongst children and adolescents. Brain Injury, 17(1), 1-23.

Hoofien, D., Gilboa, A., Vakil, E. y Donovick, P. (2001). Traumatic brain injury (TBI) 10-20 years later: A comprehensive outcome study of psychiatric symptomatology, cognitive abilities and psychosocial functioning. Brain Injury, 15(3), 189-209.

Hoyle, R. H. y Panter, A. T. (1995). Writing about structural equation models. In Rick H. Hoyle, (Ed.), (pp. 158-176). Structural equation modeling: Concepts, issues, and applications. Thousand Oaks, CA: Sage Publications.

Hu, L. y Bentler, P. M. (1999). Cutoff criteria for fit indexes in covariance structure analysis: Conventional criteria versus. Structural Equation Modeling, 6, 1-55.

Jhonson, J. y McCown, W. (1997). Terapia familiar de los trastornos neuroconductuales. Integración de la neuropsicología y la terapia familiar. Bilbao. Desclée De Brouwer S.A.

Kolakowsky-Hayner, S.S., Miner, K.D. y Kreutzer, J. (2001). Long-term life quality and family needs after traumatic brain injury. Head Trauma Rehabilitation, 16(4), 374-385.

Kreutzer, J. (1988). Family Needs Questionnaire, Richmond, Va: Rehabilitation Research and Training Center on Severe Traumatic Brain Injury, Medical College of Virginia.

Kreutzer, J., Marwitz, J.H. y Kepler, K. (1992). Traumatic brain injury: Family response and outcome. Archives of psysical medicine and rehabilitation, 73 , 771-778.

Kreutzer, J., Serio, C. y Bergquist, M. (1994). Family needs after brain injury: A quantitative analysis. Journal of Head Trauma Rehabilitation, 9(3), 104 115.

Leske, J. (1986). Needs of relatives of critically ill patients: A follow-up. Heart and Lung, 15, 189-193.

López de Arroyabe, E. (2006). Impacto del daño cerebral adquirido en las familias: necesidades, estrategias de afrontamiento y sintomas de estrés. Tesis Doctoral no publicada. Universidad de Deusto, Bilbao.

Masel, B. y Dewitt, D. (2010). Traumatic Brain Injury: A Disease Process, Not an Event. Journal of Neurotrauma, 27(8), 1529-1540.

Mathis, M. (1984). Personal needs of family members of critically ill patients with and without brain injury. Journal of Neurosurgical Nursing, 16, 36-44.

Mauss-Clum, N. y Ryan, M. (1981). Brain injury and the family. Journal of Neurosurgical Nursing, 13, 165-169.

Molter, N.C. (1979). Needs of relatives of critically ill patients: A descriptive study. Heart and Lung, 8, 332-337.

Muñoz Céspedes, J.M., Paúl, N., Pelegrín, C. y Tirapu, J. (2001). Factores pronóstico en los Traumatismos Cráneoencefálicos. Revista de Neurología, 32(4), 351-364.

Norris, L.D. y Grove, S.K. (1986). Investigation of selected psychosocial needs of family members of critically ill adults patients. Heart and Lung, 15, 194-199.

Oyserman D., Coon H. y Kemmelmeier M. (2002). Rethinking individualism and collectivism: Evaluation of theoretical assumptions and metaanalyses. Psychological Bulletin, 128, 3-73.

Ponsford, J., Sloan, S. y Snow, P. (1995). Traumatic brain injury: Rehabilitation for everyday adaptative living. Lawrence Erlbaum Associates, East Sussex.

Rokach, A., Moya, M., Orzeck, T. y Exposito, F. (2001). Loneliness in North America and Spain. Social Behavior and Personality, 29, 477-90.

Serio, C. y Kreutzer, J.S. (1992). A factor analytic study of the Family Needs Questionnaire. Comunicación presentada en la Annual Conference on the Re- 
habilitation of the Severely Brain Injured Adult and Child. Williamsburg, Va.

Serio, C., Kreutzer, J. y Gervasio, A. (1995). Predicting family needs after brain injury: Implications for intervention. Journal of Head Trauma Rehabilitation, 10(2), 32-45.

Serio, C., Kreutzer, J. y Witol, A. (1997). Family needs after traumatic brain injury: a factor analytic study of the Family Needs Questionnaire. Brain Injury, 11(1), 1-9.

Thomsen, I.V. (1984). Late outcome of severe blunt head trauma. A 10-15 year second follow up. Journal of Neurology, Neurosurgery and Psychiatry, 47, $260-268$

Triandis, HC. (1995). Individualism and collectivism. San Francisco, CA: Westview Press.

Uchino, B. N. (2004). Social support and physical healtb: Understanding the health consequences of relationships. New Haven, CT: Yale University Press.
Verhaeghe, S., Delfloor, T. y Grypdonck, M. (2005). Stress and coping among families of patients with traumatic brain injury: a review of the literature. Journal of Clinical Nursing, 14(8), 1004-1012.

Wade, S.L., Taylor, H.G., Drotar, D., Stancin, T., Yeates, K.O. y Minich, B. (2002). A prospective study of long-term caregiver and family adaptation following brain injury in children. Journal of Head Trauma Rehabilitation, 17(2), 96-111.

Witol, A., Sander, A. y Kreutzer, J. (1996). A longitudinal analysis of family needs following traumatic brain injury. Neurorehabilitation, 7, 175-187.

Wortman, C. (1984). Social support and the cancer patient: conceptual and methodological issues. Cancer, 53(2), 2339-2360.

(Articulo recibido: 20-12-2010, revisado: 30-11-2011, aceptado: 04-12-2011) 